\title{
Association between rotavirus gastroenteritis and intussusception: suggested evidence from a retrospective study in claims databases in the United States. Corresponding author: Corinne Willame, MPH, GSK, 20 Avenue Fleming, Wavre, Belgium
}

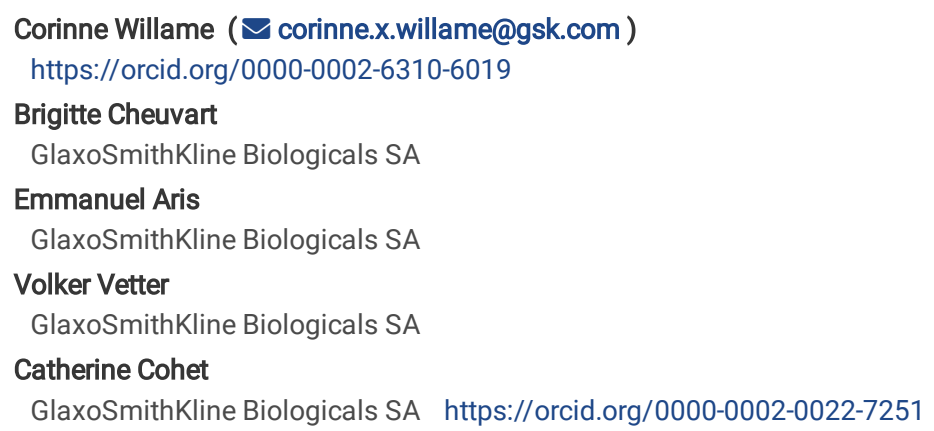




\section{Abstract}

Background: The etiology of intussusception (IS), a serious medical condition of acute gastrointestinal obstruction, remains unclear. Limited evidence suggests a role for viral infections, including rotavirus infection. This study aimed to explore the risk of IS after rotavirus gastroenteritis (RV GE) in the first year of life, where the incidence of IS is highest.

Methods: In this retrospective, self-controlled case series (SCCS), we assessed the risk of IS after RV GE in infants $<1$ year of age, using data extracted from administrative claims databases in the United States. Incidence rate ratios (IRR) of IS were calculated for the 7- and 21-day risk periods after RV GE (main analysis) or after fracture (sensitivity analysis using a control event) in subjects presenting with claims for these conditions. Post-hoc analyses were also performed.

Results: Out of the 290,912,068 subjects screened, 42 subjects presented claims for RV GE and IS (RV GE group) and 66 presented claims for fracture and IS (fracture group). The IRR of IS after RV GE was 79.6 (95\% confidence interval, Cl: 38.6-164.4) in the 7-day risk period and 25.5 (95\% Cl: 13.2-49.2) in the 21day risk period. The sensitivity analysis also showed an association between IS and fracture for the two risk periods: IRR was 6.1 (95\% $\mathrm{Cl}$ : $3.0-12.7$ ) and 2.8 (95\% Cl: $1.5-5.4)$ in the 7- and 21-day risk periods, respectively, which suggested potential confounding such as by history of rotavirus vaccination, or a visit effect. Post-hoc analyses investigating these points did not confirm an association between fracture and IS, but still suggested a possible association between RV GE and IS.

Conclusions: A temporal association between RV GE and IS was detected using the SCCS design in United States claims databases. However, due to some limitations identified through additional analyses, further studies are needed to confirm this association.

\section{Background}

Intussusception (IS) is a serious, potentially life-threatening medical condition of acute intestinal obstruction. It occurs when a segment of the bowel prolapses into a more distal portion, which can cause intestinal ischemia, infarction and perforation (1).

Between $60 \%-70 \%$ of children diagnosed with IS are below one year of age, with most episodes occurring between 5 and 7 months of age (2). IS affects mostly healthy infants and presents a male to female ratio of 3:2 (3). Estimates of IS incidence vary broadly across geographical regions, with the highest rates observed in Asia (2). Incidence rates in Europe and United States (US) are consistently similar, ranging from 20 to 66 cases per 100,000 person-years (2, $4,5)$.

To date, the etiology of IS remains unclear. Studies assessing potential risk factors for IS point towards gastroenteritis and viral infections (6). While an association with adenovirus infection has been demonstrated $(7,8)$, evidence of an association with rotavirus (RV) infection is still limited (8-10).

The implementation of RV vaccination in national immunization programs across the world has resulted in effective and substantial reductions in mortality and morbidity associated to RV GE (rotavirus gastroenteritis) (11). However, the use of live-attenuated RV vaccines at a large scale demonstrated an increased risk of IS during the first week after immunization: a meta-analysis estimated the relative risk of IS after the first dose of both live-attenuated RV vaccines as 5.4 and 5.5, and the risk after the second dose as 1.7 and 1.8 (12). Because the currently used RV vaccines are live-attenuated and replicate in the gut, the observed association between RV vaccination and IS supports the potential role of natural RV infection in the development of IS. However, to date, there is no robust evidence available on the risk of IS following RV GE.

In this retrospective study, we explored the association between RV GE and IS in children $<1$ year of age using data extracted from administrative claims databases in the United States of America (US). The aim of the study was to measure and compare the incidence of IS between pre-defined risk and control periods by applying the self-controlled case series design (SCCS), a case-only method with high sensitivity that has been largely used to assess the association between RV vaccination and IS (12-14). The risk of IS was assessed for periods of 7 and 21 days following an RV GE episode.

A summary contextualizing the outcomes of this study is displayed in the Focus on the Patient Section (Figure 1) for the information of health care professionals.

\section{Methods}

\section{Data sources and study population}

This retrospective, observational, SCCS analyzed data from two US insurance claims databases: Truven Health MarketScan ("MarketScan", consisting of Commercial Claims and Encounter databases and Medicaid databases) and Optum Clinformatics Data Mart ("Optum"). These administrative databases contain individual-level anonymized data such as socio-demographic information, clinical diagnosis, drug prescription and procedures for in- and outpatients across the US who are covered by fee-for-services and managed care plans $(15,16)$. MarketScan contains data of $>240$ million unique patients since 1995 , whereas Optum contains data of $>13$ million unique patients annually since 2000 .

The study included individual-level data in the following periods during which GSK had a license to access the data: January 2003 to December 2017 for MarketScan, and from May 2000 to December 2017 for Optum.

The primary objective was to assess the risk of IS during the 7-day period following RV GE in subjects $<1$ year of age. The secondary objective was to assess the risk of IS during the 21-day period following RV GE in the same population. These risk periods are now universally accepted in studies of the risk of RV 
vaccination using the SCCS design (12). Sensitivity analyses were conducted for fracture, a control medical event for which no plausible biological relationship with IS exists, hence used as an indicator of the quality of the data sources.

\section{Subjects, exposure and outcomes}

Subjects below one year of age who remained in a health care plan until the age of one year and for whom both RV GE or fracture claims, and claims for IS, were reported while on this plan between May 2000 and December 2017, were included.

Eligible subjects were classified into two groups: 1) RV GE group: subjects $<1$ year of age with an International Classification of Diseases (ICD) code for RV GE (ICD-10: A08.0; ICD-9: 008.61) and an ICD code for IS (ICD-10: K56.1; ICD-9: 560.0); and 2) fracture group: subjects <1 year of age with an ICD code for fracture except for clavicle fracture and fracture at birth (code list available upon request) and an ICD code for IS.

All subjects with a RV vaccination claim on the same day than a RV GE claim were excluded from the RV GE group, since it is unlikely that a child with gastroenteritis symptoms receives a RV vaccine, or that RV vaccination would trigger RV GE on the same day. RV vaccination claims were identified through current procedural terminology (CPT) codes 90680 or 90681 or national drug codes (NCD) 00006404701, 00006404720, 00006404731, 00006404741, $00008256201,58160080501,58160080502,58160080511,58160085101,58160085110,58160085302$ or 58160085452.

Claims for clavicle fracture and fracture at birth (i.e. fractures with a description containing the term "birth", "clavicle", or "clavicule") were excluded from the fracture group since they are frequently reported in newborns.

Subjects were first screened by identifying RV GE, fracture and IS claims in both databases. The Commercial and Medicaid databases belonging to MarketScan were screened separately but data were pooled. Since the databases only provide the year of birth, the complete date of birth (i.e. day, month and year) was defined as the date of earliest birth diagnosis code recorded, if available, the date of earliest record of medical diagnosis under insurance coverage, or the first date of insurance coverage. If the imputed date of birth did not coincide with the year of birth recorded in the databases, the subject was excluded from the analysis. Among screened subjects, those with RV GE or fracture and IS claims in their first year of life were selected. Since one episode of IS, RV GE or fracture could be associated with more than one claim, we considered that all recurring claims for a particular episode identified in a period $<30$ days corresponded to a single episode.

For each eligible subject identified, the following data were extracted from the database: year of birth, gender, date of claims (IS, RVGE and/or fracture) and date of RV vaccination (if available).

\section{Statistical methods}

For each group, the demographic characteristics, history of RV vaccination, age at IS, age at RV GE or fracture, and number of IS episodes (during both the risk and control periods) were summarized using descriptive statistics. We also calculated the total subject-weeks during the risk and control periods and the number of days between RV GE or fracture and IS (calculated as date of IS minus date of RV GE or fracture plus 1 day).

All statistical tests were two-sided with a significance level of alpha $=0.05$. Statistical analyses were performed using Statistical Analysis Software (SAS) version 9.3.

\section{Main analysis}

For the primary and secondary objectives of the study, we applied the SCCS method to data from the RV GE group (Figure 2).. We considered the day of RV GE claim as Day 1 . The risk period was defined as the period from Day 1 to Day 7 for the primary objective, and from Day 1 to Day 21 for the secondary objective. For the analysis of the primary objective, the period from Day 8 to Day 21 was considered as a buffer period not taken into account in the incidence rate ratio (IRR) calculations. The control period was defined as the period from birth until one year of age excluding the risk and buffer periods (Figure 2)..

The null hypothesis was that the incidence rate of IS during the risk period is the same as that during the control period. To test this hypothesis, we used the publicly available SCCS macros for SAS $(17,18)$. The IRR for IS was calculated as the incidence of IS during the risk period divided by the incidence of IS during the control period. The model included the IS event (present or absent) as the dependent variable, the period (risk or control) as the independent variable, and the following covariates for adjustment: age as a categorical variable (encoded as months since birth date) and RV vaccination-with a 7 day risk period after it-as a binary variable (present or absent).

Based on preliminary queries of the databases, it was estimated that approximately 40 cases of IS with a RV GE claim during the first year of age would be available for inclusion in the study. With 40 cases in the RV GE group, the study had $80 \%$ statistical power to demonstrate an association between RV GE and IS if the IRR was $\geq 6$, considering the risk and control periods defined for the primary objective and adjusting for the age effect.

\section{Sensitivity analysis}

We performed a sensitivity analysis by assessing the risk of IS following fracture, using the same methods, risk periods and assumptions used for the RV GE group (Figure 2).. 


\section{Post-hoc analyses}

Upon the unexpected observation of an increased risk of IS after fracture claims, we conducted post-hoc analyses to explore the consistency and validity of the results. We applied the same SCCS method as for RV GE group except that, in each post-hoc analysis, the following rationale and criteria were applied:

- Post-hoc analysis A: excluding cases where IS and RV GE claims, or IS and fracture claims, were reported on the same date, thus excluding Day 1 from the risk period and resulting in 6- and 20-day risk periods. The aim was to address a potential visit effect (19), which would cluster the reporting of IS and RV GE or fracture at the same medical visit (Day 1).

- Post-hoc analysis B: excluding cases where IS and RV GE claims were reported on the same date, and cases with a record of RV vaccination at any time. The aim was to control for a potential visit effect and to ensure that RV vaccination would not reflect a RV GE episode.

- Post-hoc analysis C: excluding cases where IS and RV GE claims, or IS and fracture claims, were reported on the same date, and considering the following risk periods: from Day -6 to Day -1 and from Day -21 to Day -1 (where Day -1 is considered as the day before RV GE or fracture). The aim was to test whether a similar risk of IS was observed during the period before RV GE or fracture, while still controlling for the visit effect.

\section{Results \\ Subject disposition}

A total of 290,912,068 subjects were screened over the period January 2003-December 2017 in MarketScan and May 2000-December 2017 in Optum. From these, 42 subjects presented claims for RV GE and IS (RV GE group) and 66 presented claims for fracture and IS (fracture group) in the first year of life (Figure 3).. There were no instances of both a RV GE code and a fracture code identified for a single subject.

\section{Descriptive data}

Table 1 shows demographic and epidemiological characteristics of the subjects. In the RV GE group, 71.4\% subjects had a birth diagnosis recorded. Subjects in the RV GE group presented claims for RV GE at a mean age of 25.2 weeks, and for IS at 26.7 weeks. While 9 (20.9\%) claims of IS occurred before RV GE, 25 (58.1\%) claims of IS occurred in the 7-day period after RV GE, and 2 (4.7\%) occurred between Day 8 and 21 after RV GE (Table 1).. When we analyzed the distribution of IS claims over the Day 1-Day 21 risk period, we found most IS claims recorded on the day of RV GE (i.e. Day 1 ), followed by Days 2 and 3 (Figure 4)..

Demographic characteristics in the fracture group were similar to those in the RV GE group, except for a slightly higher percentage of males (Table 1).. The mean ages at IS and at fracture were similar to those observed in the RV GE group for IS and RV GE; however, most of the IS claims occurred before fracture (n: $33 ; 47.1 \%$ of the cases) or in the period more than 21 days after fracture (n: 25; 35.7\%). Considering the Day 1 -Day 21 risk period, the day with most IS claims recorded was the day of fracture (i.e. Day 1) (Figure 4)..

We then analyzed the temporal distribution of RV GE, IS and RV vaccination claims during the entire first year of life of subjects in the RV GE group. Both the frequencies of RV GE and IS partially overlapped and peaked at month 3, 8 and 10. (Figure 5).. We observed 3 peaks of RV vaccination: at 2, 4 and 6 months, as expected according to the RV vaccination schedule.

In the fracture group, the frequency of fracture was stable across ages, whereas IS peaked at months 3-5 and month 9 . The temporal distribution of RV vaccination was similar to that observed in the RV GE group.

\section{Outcome data, main results and additional analyses}

The IRR of IS after RV GE was 79.6 (95\% Cl: 38.6-164.4; $\mathrm{p}<0.0001)$ in the 7-day risk period and decreased to 25.5 (13.2-49.2; $\mathrm{p}<0.0001)$ in the 21 -day risk period (Table 2).. The sensitivity analysis showed an association between IS and fracture for the two risk periods: IRR was 6.1 (3.0-12.7; $<<0.0001)$ and 2.8 (1.5-5.4; p: 0.0018) in the 7- and 21-day risk periods, respectively (Table 2)..

Post-hoc analyses (see details in Methods) are shown in Table 2. In post-hoc analysis A, after excluding Day 1 from the analysis, there was still a statistically significant association between RV GE and IS for both risk periods, but of a lower magnitude, (Table 2).. In the fracture group, there was still an association for the 6-day period but no association was detected for the 20-day period (Table 2)..

In post-hoc analysis B (in the RV GE group only), after excluding both Day 1 and cases with a recorded RV vaccination, the magnitude of the resulting IRRs was smaller but the association between RV GE and IS remained statistically significant (Table 2)..

In post-hoc analysis C, we tested whether we observed the same risk of IS before and after RV GE or fracture. For that purpose, besides excluding again the cases with IS and RV GE or fracture reported on the same date, we defined risk periods before RV GE or fracture. The results showed no statistically significant association between IS and RV GE or fracture when IS occurred before RV GE or before fracture (Table 2)..

\section{Discussion}

Main findings 
The aim of this retrospective SCCS was to explore the association between RV GE and the development of IS in infants $<1$ year of age in US claims databases.

An increased risk of IS after RV GE was observed in the main analysis. However, the sensitivity analysis also showed an increased risk of IS after fracture-an event for which there is no plausible clinical association with IS-although the magnitude of the risk was lower. Post-hoc analyses conducted to further explore the validity of the data still suggested a possible association between RV GE and IS, but not between fracture and IS.

To our knowledge, this is the first study using claims data to evaluate the association between RV GE and IS. The few previous studies that investigated this association used different designs and data sources $(7,20)$. A prospective case-control study showed that experiencing gastroenteritis during the 30 days before an IS diagnosis was associated with IS in children aged 0-59 months (odds ratio: 11.6; 95\% Cl: 3.2-41.2; p-value<0.001) (20). Although other risk factors for gastroenteritis were not explored in that study, results are consistent with other studies that identified bacterial and viral gastroenteritis as major risk factors for developing IS (21-25). While adenovirus is the primary causative agent frequently detected in IS cases, some studies demonstrated an association with rotavirus (8-10).

\section{Use of claims data}

Studies by Restivo and Bines included confirmation of IS cases by medical evaluation and/or laboratory-confirmation of the RV GE diagnosis, and collection of potential risk factor data via questionnaires (e.g. use of antibiotics, history of breastfeeding) $(7,20)$. Although such methodology is considered more robust than a retrospective database approach, the use of claims databases brings some advantages to our study. Firstly, it limits any recall bias that would be more likely present in studies relying on parents or caretaker interviews. Secondly, it allows to analyze patient-anonymized data from a large population size, which is crucial when studying events with a low background incidence such as IS (34 cases per 100,000 person-years in one study in the US) (26).

There are, however, limitations inherent to the use of claims databases. We cannot exclude a lack of validity and specificity of the IS and RV GE claims, considering that case finding for IS and RV GE was based exclusively on ICD codes declared for the reimbursement of the medical insurance claim. In the prevaccination era, RV GE ICD-9 coding was highly specific but showed less than $50 \%$ sensitivity (27). To our knowledge, there are no published studies establishing the positive predictive value (PPV) of RV GE codes in claims databases in the rotavirus post-vaccination era (i.e. after August 2006 in the US), which is when most of our data were collected. In our study, a correlation between RV GE claims and RV testing claims was not possible, since there are no corresponding laboratory data available in the databases used. In the present study, the use of insurance claims instead of electronic medical records was based on feasibility. During the design of the study, the use of the UK CPRD, a general practitioners' database which records information on RV testing was explored but then discarded due to the low number of eligible subjects available. On the other hand, regarding IS cases, we relied only on ICD codes. We used specific ICD codes to capture IS, and we excluded non-specific ICD codes (e.g. " $543.9=0$ ther and unspecified diseases of appendix"). However, this does not inform about diagnosis certainty. In two previous studies, PPVs of IS diagnosis of $84 \%$ and $49 \%$ were observed in electronic medical records and claims, respectively $(28,29)$. These values reflect the type of data sources, with higher PPVs for validated medical records such as disease registries compared to claims data.

Overall, misclassification of RV GE or IS cannot be excluded in this study. In addition, since the introduction of RV vaccination, changes in laboratory testing practices and, more generally, in clinical practice may bias the reporting of these events in claims databases. Of note, misclassification of RV GE or of IS would generally rather result in an underestimation of the association between the two events.

We observed subjects for whom RV GE and RV vaccination claims were recorded on the same day. Since the labels of the two RV vaccines available in the US recommend caution or delaying vaccination in children presenting with acute gastrointestinal disorders, it is unlikely that both RV GE and RV vaccination truly happened on the same day, suggesting that some of the RV GE claims likely reflect the indication for RV vaccination rather than a true RV GE episode. For this reason, in all analyses we excluded from the RV GE group any infant with RV GE and RV vaccination claims on the same date, and in post-hoc analysis B, we excluded any subject with a RV vaccination claim. In addition, we observed a distribution of RV vaccination claims consistent with the recommended RV vaccination schedule in the US, which points to the quality of the reporting of these claims.

In our study, IS claims were frequently reported at the same date as RV GE claims. Claims may be clustered on the same date due to a previously described phenomenon known as "Day 0 phenomenon" or "visit effect" (19). The visit effect states that collecting information only at specific time points, such as a medical visit, may cause prevalent conditions to be considered as incident, hence affecting the temporal relationship between event and outcome. To account for this effect, we conducted post-hoc analysis A, which excluded Day 1 from the analyses (thus excluding cases with claims of IS and RV GE or fracture on the same day). The reduced IRR observed in this post-hoc analysis compared to the main analysis indeed support the existence of a visit effect in the distribution of RV GE or fracture and IS claims.

Regarding potential heterogeneity between MarketScan and Optum, we did not observe any discordance between the two databases, since we obtained results consistent between data sources, and consistent with the main analysis results when data was analyzed separately for each database (data not shown).

\section{Strengths and limitations of the study design}

The SCCS method implicitly controls for time-invariant confounders, whether known or unknown. Since age could be a confounding factor for outcomes included in this study, and because RV vaccination is also age-specific, analyses were adjusted for both age and history of RV vaccination. The SCCS has been largely used to assess the association between RV vaccination and IS, and the risk periods used in the present analyses are those described in previous studies and now universally accepted (12). 
We also tested the assumption of a symmetrical pattern of IS reporting by analyzing risk periods before RV GE (post-hoc analysis C). This analysis did not detect an association between IS (occurring first) and RV GE (occurring later), which emphasizes the findings of our main analysis pointing towards a possible association between RV GE and IS in a sequential manner.

\section{Quality indicator group findings}

The main strength of our design is the inclusion of a quality indicator group that considers a medical event, fracture, for which no known nor biologically plausible association with IS exists. The aim of this sensitivity analysis was to provide information about the validity of the claims data, and indeed the results suggest a potential quality issue since a positive temporal association was observed between fracture and IS. The lower-but still significantassociation detected in the fracture group through our sensitivity analysis indicates that there is a part of spuriousness in the data, but which is difficult to quantify. To partly address this issue, we also excluded cases with claims of IS and fracture on the same date in the fracture group, in order to mitigate a potential visit effect (post-hoc analysis A). When these cases were excluded, the risk of IS after fracture further decreased or disappeared depending on the risk period considered, which could point to a visit effect in the fracture group as well.

The present study is more likely to have investigated medical encounters due to disease symptoms rather than true disease onsets. The use of a broader study endpoint (e.g. all-cause gastroenteritis) as in other studies $(6,20)$ may have limited potential outcome misclassification and could have increased sensitivity, but would not have answered our research question regarding the role of rotavirus-specific gastroenteritis in the etiology of IS. In general, the interpretation of the results of this study should take into account the nature of claims data, which are a basis for payments and are not necessarily reflecting the actual disease onset.

\section{Conclusions}

We observed a temporal positive association between RV GE and IS in US insurance claims databases. However, because of its limitations, this study should be considered as a preliminary investigation. Prospective studies and studies using validated electronic medical records are needed to confirm and quantify more precisely the association between RV GE and IS.

\section{Abbreviations}

$\mathrm{Cl}$

confidence interval

CPT

current procedural terminology

HIPAA

Health Insurance Portability and Accountability Act

ICD

International Classification of Diseases

$\mathrm{ICH}$

International Conference on Harmonization

IRR

incidence rate ratio

IS

intussusception

NCD

PPV

national drug codes

positive predictive value

RV

rotavirus 
RV GE

rotavirus gastroenteritis

SAS

Statistical Analysis Software

sccs

self-controlled case series

US

United States of America

\section{Declarations}

\section{Ethics approval and consent to participate}

This study was designed and conducted according to the principles of the Declaration of Helsinki, Good Epidemiological Practices, and the International Conference on Harmonization (ICH) guidelines for clinical investigation of medicinal products in the pediatric population (ICH E11). Retrospective data from Truven Health MarketScan Commercial Claims and Encounters and from Optum Clinformatics Data Mart are anonymized and comply with the Final Privacy Rule of the Health Insurance Portability and Accountability Act (HIPAA) of 1996. Therefore, subject informed consent and Institutional Review Board approval are not required for studies using these data sources.

\section{Consent for publication}

Not applicable.

\section{Availability of data and material}

The data that support the findings of this study are available from Truven Health MarketScan Commercial Claims and Encounters and from Optum Clinformatics Data Mart, but restrictions apply to the availability of these data. The data were used under license and so are not publicly available.

\section{Competing interests}

$\mathrm{BC}, \mathrm{CW}, \mathrm{EA}, \mathrm{VV}$ and $\mathrm{CC}$ are employed by and hold shares in the GSK group of companies.

\section{Funding}

This work was fully supported by GlaxoSmithKline Biologicals SA, which was the sole funding source and was involved in all stages of study conduct, analysis and interpretation. GlaxoSmithKline Biologicals SA took responsibility for all costs associated with the development and publishing of the present manuscript.

\section{Authors' contributions}

VV proposed the research question. CW, EA and CC designed the study. EA accessed the databases and performed the data extraction. BC conducted the statistical analyses. CW, EA, BC and CC interpreted the results. All authors critically reviewed the manuscript for important intellectual content.

\section{Acknowledgements}

\section{The authors would also like to thank Vincent Bauchau, Paola Pirrotta and Tina Singh for the support provided during the study design and/or results interpretation.}

The authors also thank Emmanuelle Ghys (Modis, Belgium c/o GSK) for publication management and Sara Rubio (Modis, Belgium c/o GSK) for providing medical writing support.

\section{References}

1.Bines JE, Kohl KS, Forster J, Zanardi LR, Davis RL, Hansen J, et al. Acute intussusception in infants and children as an adverse event following immunization: case definition and guidelines of data collection, analysis, and presentation. Vaccine. 2004;22(5-6):569-74. 
2.Jiang J, Jiang B, Parashar U, Nguyen T, Bines J, Patel MM. Childhood intussusception: a literature review. PLoS One. 2013;8(7):e68482. PubMed PMID: 23894308. Pubmed Central PMCID: PMC3718796. Epub 2013/07/31. eng.

3.Mandeville K, Chien M, Willyerd FA, Mandell G, Hostetler MA, Bulloch B. Intussusception: clinical presentations and imaging characteristics. Pediatr Emerg Care. 2012;28(9):842-4. PubMed PMID: 22929138. Epub 2012/08/30. eng.

4.Tate JE, Simonsen L, Viboud C, Steiner C, Patel MM, Curns AT, et al. Trends in intussusception hospitalizations among US infants, 1993-2004: implications for monitoring the safety of the new rotavirus vaccination program. Pediatrics. 2008;121(5):e1125-32. PubMed PMID: 18450856. Pubmed Central PMCID: PMC2680116. Epub 2008/05/03. eng.

5.Clark AD, Hasso-Agopsowicz M, Kraus MW, Stockdale LK, Sanderson CFB, Parashar UD, et al. Update on the global epidemiology of intussusception: a systematic review of incidence rates, age distributions and case-fatality ratios among children aged $<5$ years, before the introduction of rotavirus vaccination. Int J Epidemiol. 2019. PubMed PMID: 30879038.

6.Fotso Kamdem A, Vidal C, Pazart L, Leroux F, Pugin A, Savet C, et al. A case-control study of risk factors for intussusception among infants in eastern France after the introduction of the rotavirus vaccine. Vaccine. 2019. PubMed PMID: 30851968. Epub 2019/03/11. eng.

7.Bines JE, Liem NT, Justice FA, Son TN, Kirkwood CD, de Campo M, et al. Risk factors for intussusception in infants in Vietnam and Australia: adenovirus implicated, but not rotavirus. J Pediatr. 2006;149(4):452-60. PubMed PMID: 17011313. Epub 2006/10/03. eng.

8.Mansour AM, El Koutby M, El Barbary MM, Mohamed W, Shehata S, El Mohammady H, et al. Enteric viral infections as potential risk factors for intussusception. J Infect Dev Ctries. 2013;7(1):28-35. PubMed PMID: 23324817. Epub 2013/01/18. eng.

9.Konno T, Suzuki H, Kutsuzawa T, Imai A, Katsushima N, Sakamoto M, et al. Human rotavirus infection in infants and young children with intussusception. J Med Virol. 1978;2(3):265-69. PubMed PMID: 212529. Epub 1978/01/01. eng.

10.Minney-Smith CA, Levy A, Hodge M, Jacoby P, Williams SH, Carcione D, et al. Intussusception is associated with the detection of adenovirus C, enterovirus B and rotavirus in a rotavirus vaccinated population. J Clin Virol. 2014;61(4):579-84. PubMed PMID: 25464971. Epub 2014/12/04. eng.

11.Jonesteller CL, Burnett E, Yen C, Tate JE, Parashar UD. Effectiveness of Rotavirus Vaccination: A Systematic Review of the First Decade of Global Postlicensure Data, 2006-2016. Clin Infect Dis. 2017;65(5):840-50. PubMed PMID: 28444323. Epub 2017/04/27. eng.

12.Rosillon D, Buyse H, Friedland LR, Ng SP, Velazquez FR, Breuer T. Risk of Intussusception After Rotavirus Vaccination: Meta-analysis of Postlicensure Studies. Pediatr Infect Dis J. 2015;34(7):763-8. PubMed PMID: 26069948.

\section{Farrington CP. Control without separate controls: evaluation of vaccine safety using case-only methods. Vaccine. 2004;22(15-16):2064-70. PubMed PMID: 15121324.}

14.Weldeselassie YG, Whitaker HJ, Farrington CP. Use of the self-controlled case-series method in vaccine safety studies: review and recommendations for best practice. Epidemiol Infect. 2011;139(12):1805-17. PubMed PMID: 21849099.

15.Hansen L. The Truven Health MarketScan Databases for life sciences researchers; 2017. https://truvenhealth.com/Portals/0/Assets/2017-MarketScan-

Databases-Life-Sciences-Researchers-WP.pdf. Accessed 31 January 2019.

16.Optum. Optum Clinformatics Data Mart; 2014. https://www.optum.com/content/dam/optum/resources/productSheets/Clinformatics_for_Data_Mart.pe Accessed 31 January 2019.

\section{Using SAS for self-controlled case series studies; 2018. http://sccs-studies.info/sas.html. Accessed 31 January 2019.}

\section{Whitaker HJ, Farrington CP, Spiessens B, Musonda P. Tutorial in biostatistics: the self-controlled case series method. Stat Med. 2006;25(10):1768-97. PubMed PMID: 16220518. Epub 2005/10/13. eng.}

19.Jacobsen SJ, Sy LS, Ackerson BK, Chao CR, Slezak JM, Cheetham TC, et al. An unmasking phenomenon in an observational post-licensure safety study of adolescent girls and young women. Vaccine. 2012;30(31):4585-7. PubMed PMID: 22580356. Epub 2012/05/15. eng.

20.Restivo V, Costantino C, Giorgianni G, Cuccia M, Tramuto F, Corsello G, et al. Case-control study on intestinal intussusception: implications for anti-rotavirus vaccination. Expert Rev Vaccines. 2018;17(12):1135-41. PubMed PMID: 30407079.

21.Restivo V, Costantino C, Tramuto F, Vitale F. Hospitalization rates for intussusception in children aged 0-59 months from 2009 to 2014 in Italy. Hum Vaccin Immunother. 2017;13(2):445-9. PubMed PMID: 28075671. 
22.Nylund CM, Denson LA, Noel JM. Bacterial enteritis as a risk factor for childhood intussusception: a retrospective cohort study. J Pediatr. 2010;156(5):761-

5. PubMed PMID: 20138300. Epub 2010/02/09. eng.

\section{Bhisitkul DM, Todd KM, Listernick R. Adenovirus infection and childhood intussusception. Am J Dis Child. 1992;146(11):1331-3. PubMed PMID: 1415074. Epub 1992/11/01. eng.}

24.Selvaraj G, Kirkwood C, Bines J, Buttery J. Molecular epidemiology of adenovirus isolates from patients diagnosed with intussusception in Melbourne, Australia. J Clin Microbiol. 2006;44(9):3371-3. PubMed PMID: 16954276. Pubmed Central PMCID: PMC1594688. Epub 2006/09/07. eng.

25.Robinson CG, Hernanz-Schulman M, Zhu Y, Griffin MR, Gruber W, Edwards KM. Evaluation of anatomic changes in young children with natural rotavirus infection: is intussusception biologically plausible? J Infect Dis. 2004;189(8):1382-7. PubMed PMID: 15073674. Epub 2004/04/10. eng.

\section{Tate JE, Yen C, Steiner CA, Cortese MM, Parashar UD. Intussusception Rates Before and After the Introduction of Rotavirus Vaccine. Pediatrics. 2016;138(3). PubMed PMID: 27558938.}

27.Hsu VP, Staat MA, Roberts N, Thieman C, Bernstein DI, Bresee J, et al. Use of active surveillance to validate international classification of diseases code estimates of rotavirus hospitalizations in children. Pediatrics. 2005;115(1):78-82. PubMed PMID: 15629984. Epub 2005/01/05. eng.

28.Schollin Ask L, Svensson JF, Olen O, Ortqvist A. Clinical presentation of intussusception in Swedish children under 3 years of age and the validity of diagnostic coding. Pediatr Surg Int. 2019;35(3):373-81. PubMed PMID: 30478702.

29. Hoffman V, Abu-Elyazeed R, Enger C, Esposito DB, Doherty MC, Quinlan SC, et al. Safety study of live, oral human rotavirus vaccine: A cohort study in United States health insurance plans. Hum Vaccin Immunother. 2018;14(7):1782-90. PubMed PMID: 29533129. Pubmed Central PMCID: 6067866.

\section{Tables}

Table 1. Demographic and epidemiological characteristics of study subjects.

\begin{tabular}{|c|c|c|}
\hline Characteristic & $\begin{array}{c}\text { RV GE group } \\
\mathrm{N}=42\end{array}$ & $\begin{array}{l}\text { Fracture group } \\
\qquad \mathrm{N}=66\end{array}$ \\
\hline Female gender, $\mathrm{n}(\%)$ & $22(52.4)$ & $23(34.8)$ \\
\hline Birth diagnosis available, $\mathrm{n}(\%)$ & $30(71.4)$ & $48(72.7)$ \\
\hline Age in weeks at IS, mean (SD) & $26.7(13.9)$ & $24.4(12.8)$ \\
\hline Age in weeks at RV GE or fracture, mean (SD) & $25.2(14.6)$ & $24.8(15.0)$ \\
\hline \multicolumn{3}{|l|}{ IS episodes, n (\%) } \\
\hline Before event & $9(20.9)$ & $33(47.1)$ \\
\hline Day 1-Day 7 & $25(58.1)$ & $9(12.9)$ \\
\hline Day 8-Day 21 & $2(4.7)$ & $3(4.3)$ \\
\hline After Day 21 & $7(16.3)$ & $25(35.7)$ \\
\hline \multicolumn{3}{|l|}{ Subject-weeks } \\
\hline Before event & 1054.0 & 1632.1 \\
\hline Day 1-Day 7 & 42.0 & 74.6 \\
\hline Day 8-Day 21 & 84.0 & 144.3 \\
\hline After Day 21 & 857.9 & 1298.0 \\
\hline
\end{tabular}

IS, intussusception; $\mathrm{N}$, number of subjects in the group; $\mathrm{n}(\%)$, number (percentage) of subjects in a given category; RV GE, rotavirus gastroenteritis; SD, standard deviation Day 1 was defined as the day of RV GE or fracture claim.

Table 2. Incidence rate ratio of intussusception after RV GE or fracture in the main analysis (RV GE group), sensitivity analysis (fracture group) and post-hoc analyses $\mathrm{A}, \mathrm{B}$ and $\mathrm{C}$. 
Main (RV GE group) and sensitivity (fracture group) analyses

\begin{tabular}{|c|c|c|c|c|c|c|}
\hline & \multicolumn{3}{|c|}{ RV GE group, $N=42$} & \multicolumn{3}{|c|}{ Fracture group, N=66 } \\
\hline & $\mathrm{n}$ & $\begin{array}{c}\text { IRR } \\
(95 \% \mathrm{Cl})\end{array}$ & p-value & $\mathrm{n}$ & $\begin{array}{c}\text { IRR } \\
(95 \% \mathrm{Cl})\end{array}$ & p-value \\
\hline \multicolumn{7}{|l|}{ Risk period } \\
\hline Day 1-7 & 41 & $\begin{array}{c}79.6 \\
(38.6-164.4)\end{array}$ & \multirow{2}{*}{$\begin{array}{l}<0.0001 \\
<0.0001\end{array}$} & 67 & $\begin{array}{c}6.1 \\
(3.0-12.7)\end{array}$ & $<0.0001$ \\
\hline Day 1-21 & 43 & $\begin{array}{c}25.5 \\
(13.2-49.2) \\
\end{array}$ & & 70 & $\begin{array}{c}2.8 \\
(1.5-5.4) \\
\end{array}$ & 0.0018 \\
\hline \multicolumn{7}{|c|}{ Post-hoc analysis A } \\
\hline & \multicolumn{3}{|c|}{ RV GE group, N=26 } & \multicolumn{3}{|c|}{ Fracture group, $\mathbf{N}=61$} \\
\hline & $\mathrm{n}$ & $\begin{array}{c}\text { IRR } \\
(95 \% \mathrm{Cl})\end{array}$ & p-value & $\mathrm{n}$ & $\begin{array}{c}\text { IRR } \\
(95 \% \mathrm{Cl})\end{array}$ & p-value \\
\hline \multicolumn{7}{|l|}{ Risk period } \\
\hline Day 2-7 & 25 & $\begin{array}{c}29.9 \\
(12.2-73.3)\end{array}$ & \multirow{2}{*}{$\begin{array}{l}<0.0001 \\
<0.0001\end{array}$} & 62 & $\begin{array}{c}3.1 \\
(1.1-8.7)\end{array}$ & 0.0320 \\
\hline Day 2-21 & 27 & $\begin{array}{c}10.7 \\
(4.7-24.3) \\
\end{array}$ & & 65 & $\begin{array}{c}1.7 \\
(0.8-3.8) \\
\end{array}$ & 0.2049 \\
\hline \multicolumn{7}{|c|}{ Post-hoc analysis B (conducted in RV GE group only) } \\
\hline & \multicolumn{3}{|c|}{ RV GE group, N=18 } & & & \\
\hline & $\mathrm{n}$ & $\begin{array}{c}\text { IRR } \\
(95 \% \mathrm{Cl})\end{array}$ & p-value & & & \\
\hline \multicolumn{7}{|l|}{ Risk period } \\
\hline Day 2-7 & 18 & $\begin{array}{c}21.1 \\
(6.9-64.5)\end{array}$ & $<0.0001$ & & & \\
\hline Day $2-21$ & 18 & $\begin{array}{c}5.8 \\
(2.0-17.4) \\
\end{array}$ & 0.0016 & & & \\
\hline \multicolumn{7}{|c|}{ Post-hoc analysis C } \\
\hline & \multicolumn{3}{|c|}{ RV GE group, $\mathbf{N}=26$} & \multicolumn{3}{|c|}{ Fracture group, $\mathrm{N}=61$} \\
\hline \multicolumn{7}{|l|}{ Risk period } \\
\hline Day $-6--1$ & 25 & $\begin{array}{c}4.3 \\
(1.0-19.3)\end{array}$ & 0.0546 & 60 & $\begin{array}{c}0.7 \\
(0.1-5.2)\end{array}$ & 0.7449 \\
\hline Day $-21--1$ & 27 & $\begin{array}{c}2.9 \\
(0.9-8.8) \\
\end{array}$ & 0.0658 & 65 & $\begin{array}{c}1.3 \\
(0.6-3.1) \\
\end{array}$ & 0.5277 \\
\hline
\end{tabular}

IRR, incidence rate ratio (calculated as the incidence of IS during the risk period divided by the incidence of IS during the control period); IS, intussusception; $n$, number of cases that presented RV GE and IS or fracture and IS claims in the whole study period (one subject may contribute to several cases if presenting more than one RV GE or fracture claim); RV, rotavirus; RV GE, rotavirus gastroenteritis; $95 \% \mathrm{Cl}, 95 \%$ Wald confidence interval.

Day 1 was defined as the day of RV GE or fracture claim.

p-values in bold indicate statistical significance.

Post-hoc analysis A: analysis excluding cases with IS and RV GE or fracture claims on the same day (Day 1 was excluded from this analysis)

Post-hoc analysis B: analysis excluding cases with IS and RVGE claims on the same day, and cases with RV vaccination (Day 1 was excluded from this analysis)

Post-hoc analysis C: analysis excluding cases with IS and RV GE or fracture claims on the same day and considering risk period before RV GE or fracture (Day -1 is considered as the day before the RV GE claim).

\section{Figures}




\section{Focus on the Patient}

\section{What is the context?}

Intussusception is a rare intestinal obstruction occurring in young infants. It can be life-threatening if not timely diagnosed and treated.

Rotavirus is known to be one of the leading causes of diarrheal diseases. Some limited data suggest that rotavirus infection could be associated with a risk of intussusception.

\section{What is new?}

We analyzed United States claims data to evaluate the risk of intussusception after rotavirus gastroenteritis in children below one year of age. We validated our observations by investigating if this risk was also observed after an unrelated control medical event (fracture).

We observed that rotavirus gastroenteritis increases the risk of developing intussusception within a short timeframe after infection. A similar association between fracture and intussusception was not shown.

\section{What is this important?}

This analysis of real-world data points towards a role of rotavirus infection in the development of intussusception. Additional studies are needed to confirm and better quantify this association.

Figure 1

Focus on the Patient Section

7-day risk period

\begin{tabular}{|l|l|l|}
\hline & & \\
\hline
\end{tabular}

21-day risk period
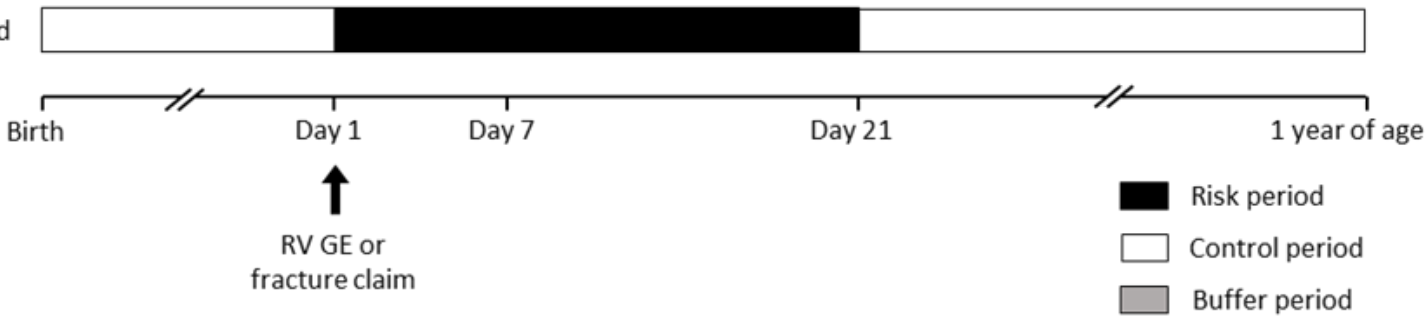

Figure 2

Study design. RV GE, rotavirus gastroenteritis. Day 1 was defined as the day of RV GE or fracture claim. The buffer period was not taken into consideration in the analyses. 


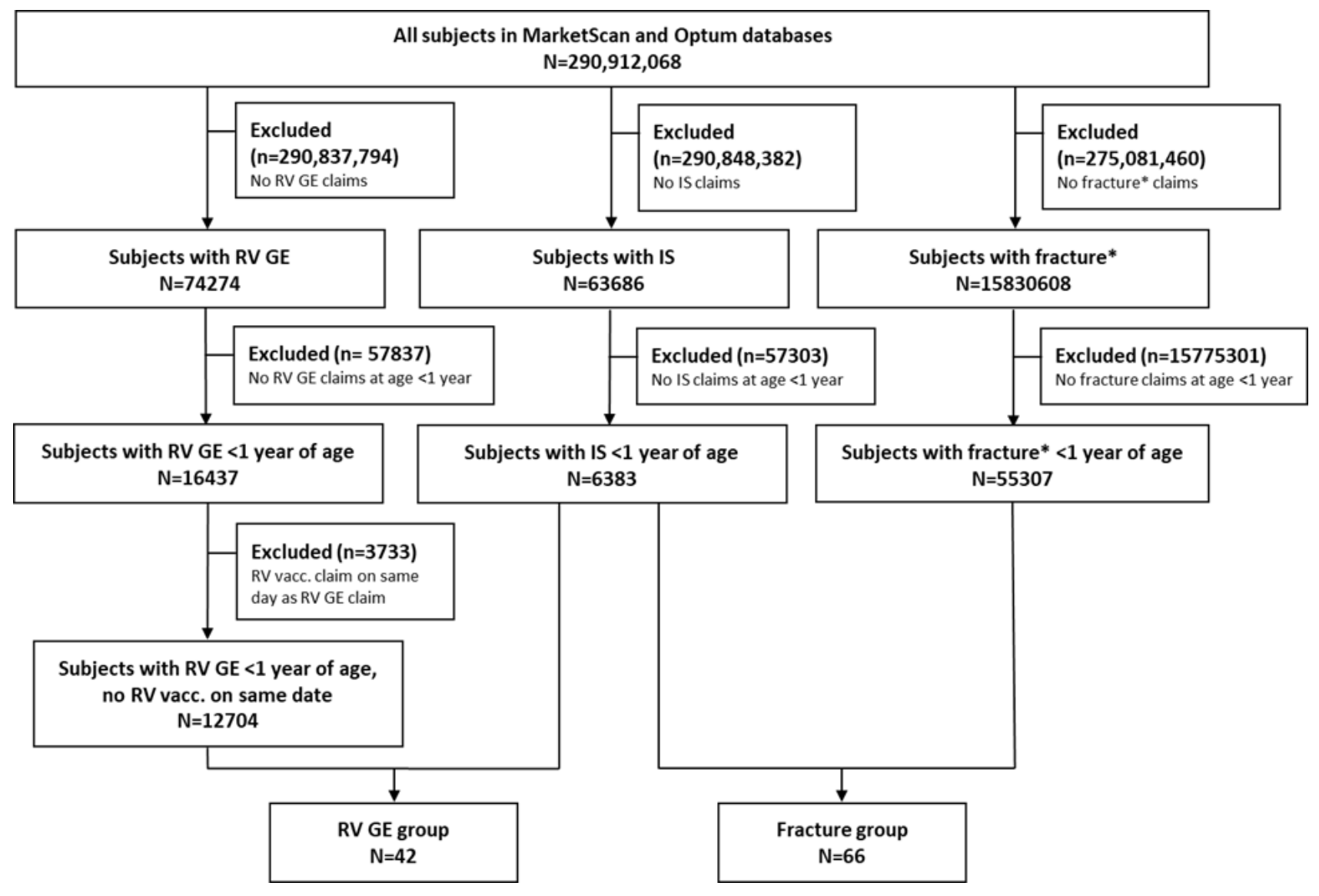

Figure 3

Disposition of subjects in the study. IS, intussusception; N, number of subjects; n, number of subjects excluded (with reason for exclusion); RV GE, rotavirus gastroenteritis; RV vacc., rotavirus vaccination. * Clavicle fracture and fracture at birth were excluded from this study. 
A

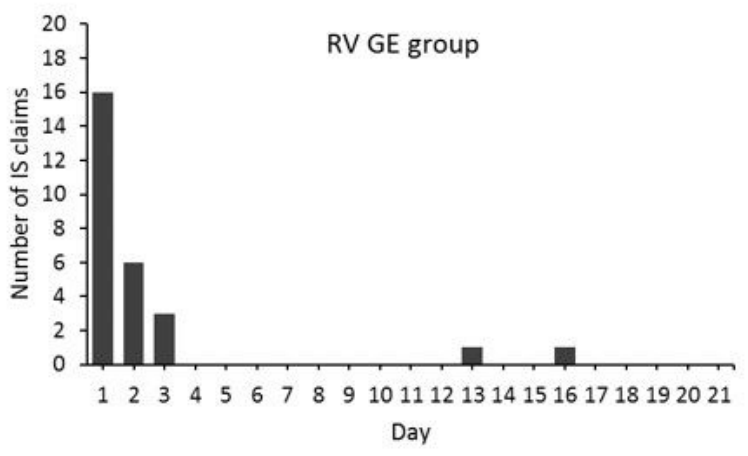

B

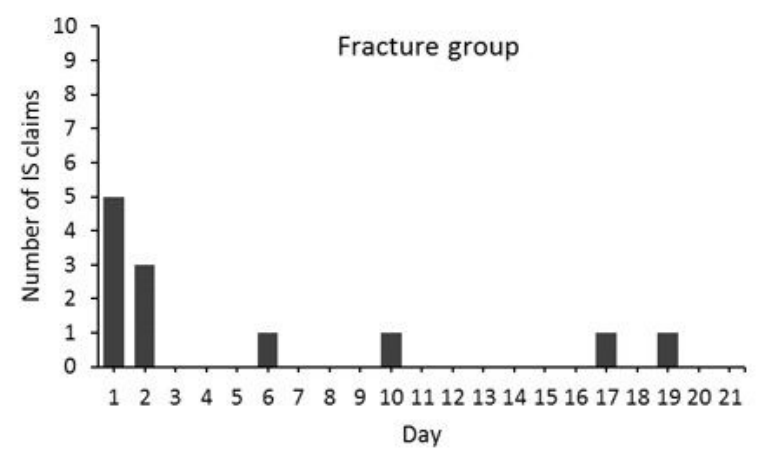

Figure 4

Distribution of intussusception claims during the 21-day risk period after RV GE (A) or fracture (B). IS, intussusception; RV GE, rotavirus gastroenteritis. Day 1 was defined as the day of RV GE or fracture claim.

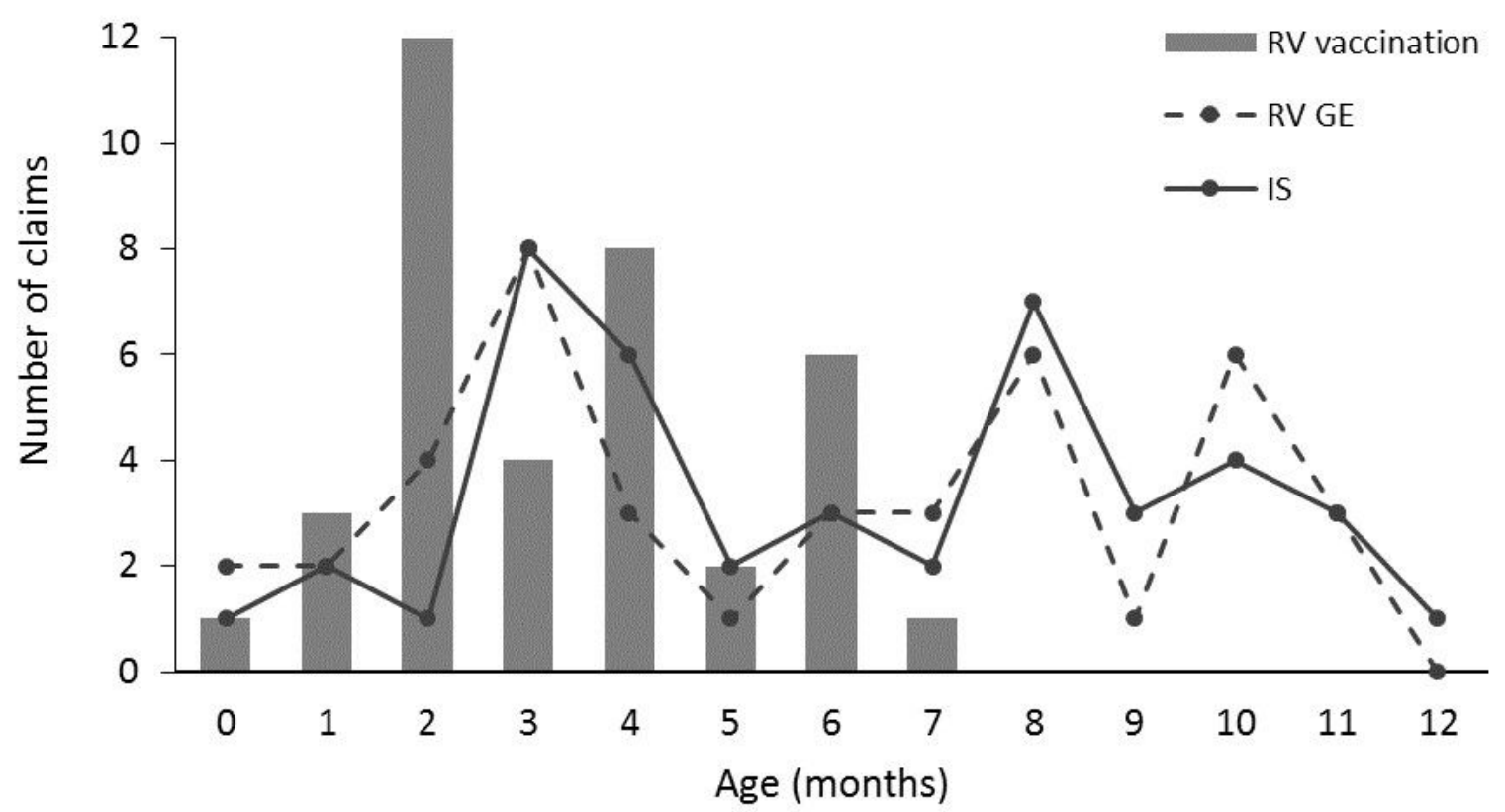

Figure 5 
Distribution of claims of rotavirus gastroenteritis, intussusception and rotavirus vaccination by age (RV GE group). IS, intussusception; RV GE, rotavirus; RV GE, rotavirus gastroenteritis. 